Article

\title{
Synthesis and Antimicrobial Activity of Some New 1,3,4-Thiadiazole Derivatives
}

\author{
Thoraya A. Farghaly, Magda A. Abdallah * and Mohamed R. Abdel Aziz \\ Department of Chemistry, Faculty of Science, Cairo University, Giza 12613, Egypt \\ * Author to whom correspondence should be addressed; E-Mail: mkamalh2009@yahoo.com.
}

Received: 16 October 2012; in revised form: 23 November 2012 / Accepted: 26 November 2012 / Published: 10 December 2012

\begin{abstract}
New series of 1,3,4-thiadiazoles have been prepared via reaction of 1,3,4-thiadiazolenaminones $\mathbf{1}$ with $N$-phenyl 2-oxopropanehydrazonoyl chloride (2) in dioxane in the presence of triethylamine. Also, some new heterocycles incorporating 1,3,4-thiadiazole ring were obtained by reaction of 1,3,4-thiadiazolenaminones $\mathbf{1}$ with nitrogen-nucleophiles like hydrazine hydrate, 3-amino-1,2,4-triazole and 2-aminobenzimidazole. The structure of the new products was established based on elemental and spectral analysis. The relation between the structure of the products and their activity towards some microorganisms was studied and promising results were obtained.
\end{abstract}

Keywords: antimicrobial activity; enaminone; 1,3,4-thiadiazole; hydrazonoyl halides

\section{Introduction}

Recently, the chemistry of enaminones has received considerable attention due to their utility as building blocks in heterocyclic synthesis [1-5]. On the other hand, 1,3,4-thiadiazole derivatives have attracted considerable interest owing to their wide spectrum of biological activity, including anti-microbial, anti-tuberculosis, anticonvulsant, anti-inflammatory, and antiulcer properties [6-10]. Recently, we published the antimicrobial activity results of a series of $\mathrm{N}$-[3-aryl-5-(3-dimethylaminoacryloyl)-3H-[1,3,4]-thiadiazol-2-ylidene]-benzamides, which showed promising activity [11]. Based on these findings, and in continuation of our interest in synthesis of bioactive compounds [12-16], we have now prepared a new series of 1,3,4-thiadiazoles via reaction of $\mathrm{N}$-[3-aryl-5-(3-dimethylaminoacryloyl)-3H-[1,3,4]-thiadiazol-2-ylidene]-benzamides with 1,3-dipoles and some nitrogen 
nucleophiles to investigate the antimicrobial activity of the products and study their structure activity relationship (SAR) towards some microorganisms.

\section{Results and Discussion}

Recently, 1,3,4-thiadiazole-enaminones 1a-d were prepared in our laboratory via reaction of 5-acetyl-3-aryl-2-benzoylimino-1,3,4-thiadiazoles with dimethylformamide-dimethylacetal (DMF-DMA) under reflux in dry toluene [11]. The reactions of these enaminones as dipolarophiles with 1,3-dipoles were studied next. Thus, reaction of $\mathbf{1 a - d}$ with hydrazonoyl chloride $\mathbf{2}$ in refluxing dioxane in the presence of triethylamine afforded, in each case, one isolable product, as evidenced by TLC analysis. The structures of the isolated products were identified, based on their elemental analyses and spectral (IR, ${ }^{1} \mathrm{H}-\mathrm{NMR},{ }^{13} \mathrm{C}-\mathrm{NMR}$ and MS) data, as the respective 3-aryl-2-benzoylimino-5-(1-phenyl-3-acetylpyrazol-4-yl-carbonyl)-1,3,4-thiadiazoles $\mathbf{4 a - d}$ (Scheme 1).

Scheme 1. Reaction of enaminones 1a-d with hydrazonoyl chloride 2.

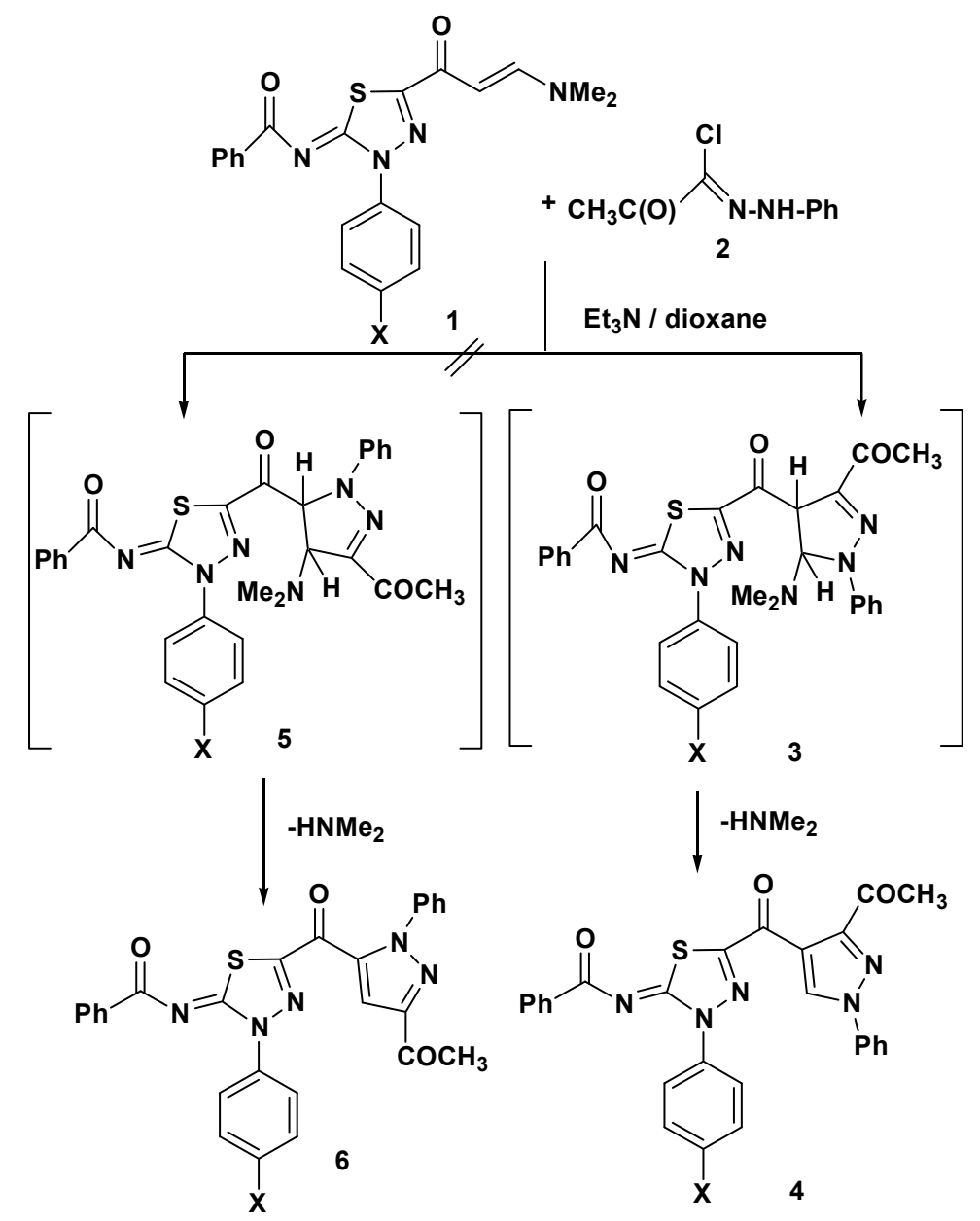

$\mathrm{X}: \mathrm{a}, \mathrm{OCH}_{3} ; \mathrm{b}, \mathrm{CH}_{3} ; \mathrm{c}, \mathrm{Cl} ; \mathrm{d}, \mathrm{NO}_{2}$

For example, the IR spectra of products 4 revealed in each case, three $\mathrm{C}=\mathrm{O}$ absorption bands in the 1694-1680, 1642-1636 and 1617-1604 $\mathrm{cm}^{-1}$ region. Also, their ${ }^{1} \mathrm{H}-\mathrm{NMR}$ spectra exhibited in each case characteristic singlet signals at 2.44-2.38 and 9.34-9.31 ppm assigned to the protons of the acetyl 
group and pyrazole- $\mathrm{CH}$, respectively. The ${ }^{13} \mathrm{C}-\mathrm{NMR}$ of compounds $\mathbf{4 a - d}$ revealed three signals at 176-172, 190-189 and 206-196 for the carbons of the three carbonyl groups. The mechanism of formation of products 4 is depicted in Scheme 1. The suggested pathway is consistent with all reports published about the reaction of hydrazonoyl halides with enaminones which indicate that this reaction leads to the formation of 5-unsubstituted pyrazole derivatives [11,17,18] and not the regioisomeric 4-unsubstituted pyrazoles 6.

The reactivity of thiadiazole-enaminones $\mathbf{1 a}-\mathbf{d}$ with some nitrogen-nucleophiles was studied next. Thus, reaction of 1a-d with 3-amino-1,2,4-triazole in acetic acid under reflux led to formation of 1,2,4-triazolo[1,5-a]pyrimidine derivatives 7 (Scheme 2). Similarly, reaction of $\mathbf{1 a - d}$ with 2-aminobenzimidazole under the same reaction conditions afforded the respective benzimidazo [1,2-a]pyrimidines 8 (Scheme 2).

Scheme 2. Reaction of enaminones 1a-d with heterocyclic amines.

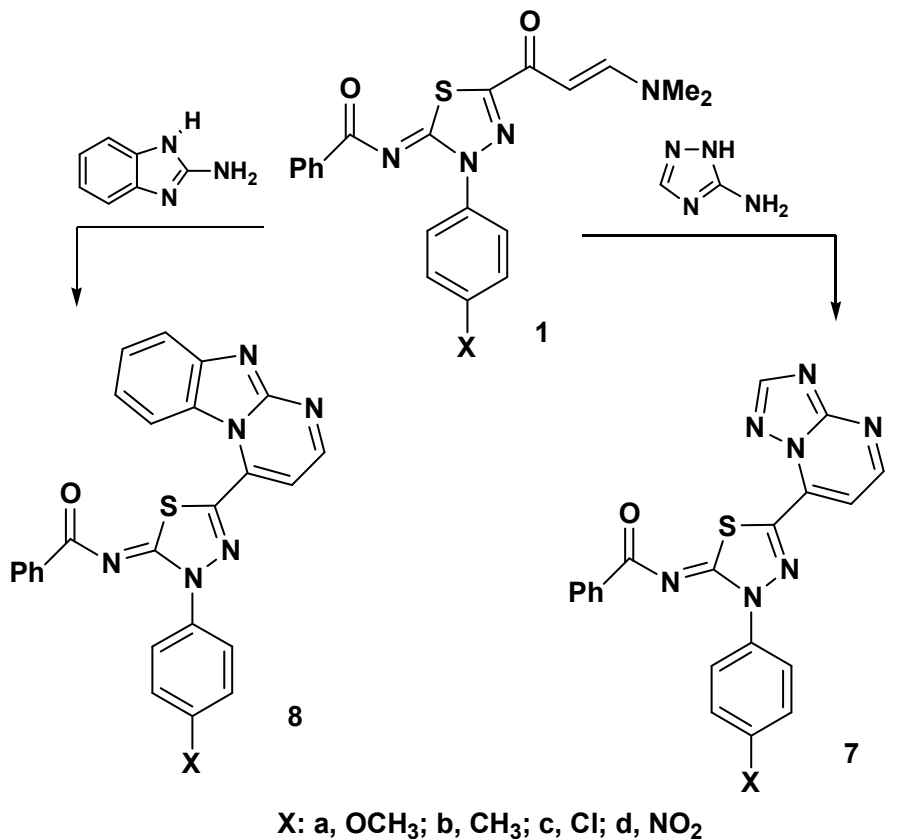

The pathway of formation of products 7 and $\mathbf{8}$ involves Michael addition of the exocyclic amino group of the heteroamines to the enaminone double bond of $\mathbf{1}$, followed by in situ tandem elimination of dimethylamine and dehydrative cyclization. The structure of the products $\mathbf{7}$ and $\mathbf{8}$ was confirmed based on elemental and spectral data (see Experimental). For example, the IR spectra of products $\mathbf{7}$ and $\mathbf{8}$ revealed in each case the absence of the carbonyl absorption band due to the enaminone residue in compounds $\mathbf{1}$. Also, the ${ }^{1} \mathrm{H}-\mathrm{NMR}$ spectrum of each of the products $\mathbf{7}$ and $\mathbf{8}$ displayed two doublets in the regions 9.71-8.14 and 8.56-8.12 ppm with $J$ values near $5 \mathrm{~Hz}$ that are assignable to the two vicinal protons in the pyrimidine moieties [19-21].

On the other hand, reaction of enaminones 1a-d with hydrazine hydrate in ethanol under reflux led to formation of the thiadiazole-pyrazole linked products 9 (Scheme 3). The structure of the latter products was established using spectroscopic and elemental analysis methods. For example, the IR spectra of products 9 revealed in each case only one carbonyl band near $1610 \mathrm{~cm}^{-1}$ attributed to the 
benzoylimino group. In addition, the ${ }^{1} \mathrm{H}-\mathrm{NMR}$ spectra of products 9 exhibited a singlet signal at $\delta 9.0-10.34 \mathrm{ppm}$ due to the $\mathrm{NH}$ proton of pyrazole ring.

Scheme 3. Reaction of enaminones 1a-d with hydrazine hydrate.

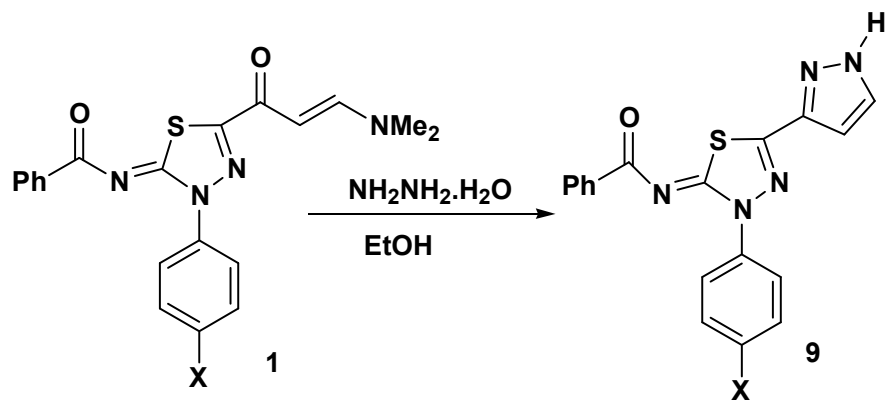

$X: a, \mathrm{OCH}_{3} ; \mathrm{b}, \mathrm{CH}_{3} ; \mathrm{c}, \mathrm{Cl} ; \mathrm{d}, \mathrm{NO}_{2}$

\subsection{Biological Screening}

\subsubsection{Antimicrobial Activity}

In vitro antimicrobial screening of compounds 4, 7, 8 and 9 prepared in this study was carried out using cultures of four fungal strains, including Aspergillus fumigatus (RCMB 002003) (AF), P. italicum (RCMB 005003, PI), Geotrichum candidum (RB 052006, GC), and Candida albicans (RCMB 005002, CA) as well as four bacteria species, namely, Gram positive bacteria, Staphylococcus aureus (RCMB 000106, SA) and Bacillus subtilis (RCMB 000107, BS), Gram negative bacteria, Pseudomonas aeruginosa (RCMB 000102, PA) and Escherichia coli (RCMB 000103, EC). Amphotericin B as an antifungal agent, ampicillin as an antibacterial agent for Gram positive bacteria and gentamicin as an antibacterial agent for Gram negative bacteria were used as references to evaluate the potency of the tested compounds under the same conditions.

\subsubsection{Antimicrobial Activity Screening and Structure Activity Relationship}

The results of antimicrobial activities for some of the newly synthesized compounds showed promising effects compared to control drugs (see Table 1). Compounds $\mathbf{4 a}$ and $\mathbf{4 b}$ have high potency as antifungals except for the fungus Candida albicans (CA). Replacing the substituent $\mathrm{X}$ in the phenyl group at position 3 of the 1,3,4-thiadiazole moiety in compounds 4 with electron withdrawing groups, e.g., $\mathrm{X}=\mathrm{Cl}, \mathrm{NO}_{2}$, as in $\mathbf{4} \mathbf{c}$ and $\mathbf{4 d}$, leads to a decrease in the antifungal activity to zero (Table 1). The results also showed that compounds $\mathbf{4 a}$ and $\mathbf{4 b}$ have high potency towards Gram positive bacteria $S A$ and $B S$ and Gram negative bacteria $P A$. Their antibacterial activity is high compared with compounds $\mathbf{4 c , d}$, which is in agreement with what was mentioned before. On the other hand, compounds $\mathbf{7 c}$ and $\mathbf{7 d}$ have higher potency against all tested fungi except $C A$ than compound $\mathbf{7 a}$. Compound $\mathbf{7 b}$ has no activity towards any of the tested fungi. In addition, compounds $7 \mathbf{a}, \mathbf{7 c}$ and $\mathbf{7 d}$ have high activity against almost all bacteria used, while compound $\mathbf{7 b}$ has no activity against any of the tested bacteria. This indicates that replacing $X$ in the phenyl group at position-3 of 1,3,4-thiadiazole moiety in compounds 7 with an electron donating group decreased the activity of these compounds towards all tested microorganisms. In addition, the results depicted in Table 1 revealed the high potency of 
compounds 8 towards all tested microorganisms, except fungus $C A$ and Gram negative bacteria $P A$. The order of decreasing reactivity towards tested fungi and bacteria is as follows: $\mathbf{8 b}>\mathbf{8 c}>\mathbf{8 a}$. Furthermore, the results of Table 1 indicated that compounds $9 \mathbf{a}$ and $\mathbf{9 b}$ have high potency towards all tested fungi except fungus $C A$ and Gram negative bacteria $P A$. The activity of compounds $\mathbf{9 a}, \mathbf{b}$ can be attributed to the presence of pyrazole ring and the small size of the molecules.

Table 1. Antimicrobial activity expressed as inhibition diameter zones in millimeter ( $\mathrm{mm})$ of compounds 4, 7, 8 and 9 against the pathological strains based on well diffusion as assay.

\begin{tabular}{|c|c|c|c|c|c|c|c|c|}
\hline \multirow{2}{*}{$\begin{array}{l}\text { Compound } \\
\text { No. }\end{array}$} & \multicolumn{4}{|c|}{ Fungi } & \multicolumn{2}{|c|}{ Gram positive bacteria } & \multicolumn{2}{|c|}{ Gram negative bacteria } \\
\hline & $\begin{array}{c}\text { A. } \\
\text { fumigatus }\end{array}$ & $\begin{array}{c}P . \\
\text { italicum }\end{array}$ & $\begin{array}{c}C . \\
\text { albicans }\end{array}$ & $\begin{array}{c}\text { G. } \\
\text { candidum }\end{array}$ & $\begin{array}{c}S . \\
\text { aureus }\end{array}$ & $\begin{array}{c}B . \\
\text { subtilis }\end{array}$ & $\begin{array}{c}P . \\
\text { aeruginosa }\end{array}$ & E. coli \\
\hline $4 a$ & $\begin{array}{l}15.6 \\
( \pm 0.19)\end{array}$ & $\begin{array}{l}16.3 \\
( \pm 0.24)\end{array}$ & N.A. & $\begin{array}{l}19.8 \\
( \pm 0.38)\end{array}$ & $\begin{array}{l}17.9 \\
( \pm 0.27)\end{array}$ & $\begin{array}{l}20.2 \\
( \pm 0.14)\end{array}$ & N.A. & $\begin{array}{l}10.9 \\
( \pm 0.29)\end{array}$ \\
\hline $4 b$ & $\begin{array}{l}19.6 \\
( \pm 0.13)\end{array}$ & $\begin{array}{l}20.3 \\
( \pm 0.22)\end{array}$ & N.A. & $\begin{array}{l}22.4 \\
( \pm 0.14)\end{array}$ & $\begin{array}{l}22.8 \\
( \pm 0.25)\end{array}$ & $\begin{array}{l}25.8 \\
( \pm 0.31)\end{array}$ & N.A. & $\begin{array}{l}17.6 \\
\pm 0.25\end{array}$ \\
\hline $4 c$ & $\begin{array}{l}10.3 \\
( \pm 0.12)\end{array}$ & N.A. & N.A. & N.A. & $\begin{array}{l}12.0 \\
( \pm 0.21)\end{array}$ & $\begin{array}{l}12.3 \\
( \pm 024)\end{array}$ & N.A. & $\begin{array}{l}8.4 \\
( \pm 0.12)\end{array}$ \\
\hline 4d & N.A. & N.A. & N.A. & N.A. & $\begin{array}{l}13.6 \\
( \pm 0.17)\end{array}$ & $\begin{array}{l}15.4 \\
( \pm 0.33)\end{array}$ & N.A. & N.A. \\
\hline $7 a$ & $\begin{array}{l}13.9 \\
( \pm 0.25)\end{array}$ & $\begin{array}{l}16.7 \\
( \pm 0.19)\end{array}$ & N.A. & $\begin{array}{l}19.8 \\
( \pm 0.35)\end{array}$ & $\begin{array}{l}11.7 \\
( \pm 0.14)\end{array}$ & $\begin{array}{l}14.6 \\
( \pm 0.67)\end{array}$ & N.A. & N.A. \\
\hline $7 b$ & N.A & N.A. & N.A. & N.A & N.A & N.A & N.A. & N.A. \\
\hline $7 \mathrm{c}$ & $\begin{array}{l}18.5 \\
( \pm 0.15)\end{array}$ & $\begin{array}{l}19.2 \\
( \pm 0.11)\end{array}$ & N.A. & $\begin{array}{l}20.9 \\
( \pm 0.26)\end{array}$ & $\begin{array}{l}18.3 \\
( \pm 0.19)\end{array}$ & $\begin{array}{l}19.2 \\
( \pm 0.21)\end{array}$ & N.A. & $\begin{array}{l}12.2 \\
( \pm 0.13)\end{array}$ \\
\hline $7 d$ & $\begin{array}{l}14.8 \\
( \pm 0.13)\end{array}$ & $\begin{array}{l}12.6 \\
( \pm 0.21)\end{array}$ & N.A. & $\begin{array}{l}16.8 \\
( \pm 0.22)\end{array}$ & $\begin{array}{l}15.0 \\
( \pm 0.18)\end{array}$ & $\begin{array}{l}11.2 \\
( \pm 0.12)\end{array}$ & N.A. & $\begin{array}{l}11.3 \\
( \pm 0.31)\end{array}$ \\
\hline $8 a$ & $\begin{array}{l}14.3 \\
( \pm 0.21)\end{array}$ & $\begin{array}{l}15.2 \\
( \pm 0.23)\end{array}$ & N.A. & $\begin{array}{l}11.7 \\
( \pm 0.15)\end{array}$ & $\begin{array}{l}15.8 \\
( \pm 0.31)\end{array}$ & $\begin{array}{l}18.6 \\
( \pm 0.21)\end{array}$ & N.A. & N.A. \\
\hline $8 b$ & $\begin{array}{l}20.4 \\
( \pm 0.19)\end{array}$ & $\begin{array}{l}21.6 \\
( \pm 0.12)\end{array}$ & N.A. & $\begin{array}{l}25.8 \\
( \pm 0.37)\end{array}$ & $\begin{array}{l}23.9 \\
( \pm 0.27)\end{array}$ & $\begin{array}{l}26.7 \\
( \pm 0.14)\end{array}$ & N.A. & $\begin{array}{l}19.8 \\
( \pm 0.10)\end{array}$ \\
\hline $8 \mathrm{c}$ & $\begin{array}{l}16.9 \\
( \pm 0.13)\end{array}$ & $\begin{array}{l}17.8 \\
( \pm 0.24)\end{array}$ & N.A. & $\begin{array}{l}21.4 \\
( \pm 0.17)\end{array}$ & $\begin{array}{l}19.4 \\
( \pm 0.27)\end{array}$ & $\begin{array}{l}20.4 \\
( \pm 0.14)\end{array}$ & N.A. & $\begin{array}{l}10.6 \\
( \pm 0.31)\end{array}$ \\
\hline 9a & $\begin{array}{l}20.3 \\
( \pm 0.31)\end{array}$ & N.A. & N.A. & $\begin{array}{l}22.6 \\
( \pm 0.22)\end{array}$ & $\begin{array}{l}23.7 \\
( \pm 0.31)\end{array}$ & $\begin{array}{l}25.9 \\
( \pm 0.22)\end{array}$ & N.A. & $\begin{array}{l}15.9 \\
( \pm 0.38)\end{array}$ \\
\hline 9b & $\begin{array}{l}21.6 \\
( \pm 0.22)\end{array}$ & $\begin{array}{l}20.8 \\
( \pm 0.12)\end{array}$ & N.A. & $\begin{array}{l}26.2 \\
( \pm 025)\end{array}$ & $\begin{array}{l}22.0 \\
( \pm 0.23)\end{array}$ & $\begin{array}{l}21.4 \\
( \pm 012)\end{array}$ & N.A. & $\begin{array}{l}18.9 \\
( \pm 0.26)\end{array}$ \\
\hline Amphotericin B & $\begin{array}{l}23.7 \\
( \pm 0.10)\end{array}$ & $\begin{array}{l}21.9 \\
( \pm 0.12)\end{array}$ & $\begin{array}{l}19.8 \\
( \pm 0.20)\end{array}$ & $\begin{array}{l}28.7 \\
( \pm 0.22)\end{array}$ & N.A. & N.A. & N.A. & N.A. \\
\hline Ampicillin & N.A. & N.A. & N.A. & N.A. & $\begin{array}{l}27.4 \\
( \pm 0.18)\end{array}$ & $\begin{array}{l}32.4 \\
( \pm 0.10)\end{array}$ & N.A. & N.A. \\
\hline Gentamicin & N.A. & N.A. & N.A. & N.A. & N.A. & N.A. & $\begin{array}{l}17.3 \\
( \pm 0.15)\end{array}$ & $\begin{array}{l}22.3 \\
( \pm 0.18)\end{array}$ \\
\hline
\end{tabular}

The experiment was carried out in triplicate and average zone of inhibition was calculated (100 $\mu \mathrm{L}$ was tested) (N.A. $=$ no activity), data are expressed in the form of mean \pm SD. 


\subsubsection{Minimum Inhibitory Concentration (MIC)}

The minimum inhibitory concentration (MIC) of the synthesized compounds against highly inhibited organisms is reported in Table 2. Compound 9 b revealed high a MIC value of $0.9 \mu \mathrm{g} / \mathrm{mL}$ against Aspergillus fumigatus (RCMB 002003), $0.08 \mu \mathrm{g} / \mathrm{mL}$ against Geotrichum candidum and $1.95 \mu \mathrm{g} / \mathrm{mL}$ against Straphylococcus aureus. Compounds $\mathbf{4 c}$ and 9a exhibited a low MIC value of $0.12 \mu \mathrm{g} / \mathrm{mL}$ against Gram positive bacteria (BS), while compound $\mathbf{8 b}$ revealed a MIC of $7.81 \mu \mathrm{g} / \mathrm{mL}$ against $P I$.

Table 2. Minimum inhibitory concentration $(\mu \mathrm{g} / \mathrm{mL})$ against the pathological strains.

\begin{tabular}{|c|c|c|c|c|c|c|c|c|}
\hline \multirow{2}{*}{$\begin{array}{l}\text { Compound } \\
\text { No. }\end{array}$} & \multicolumn{4}{|c|}{ Fungi } & \multicolumn{2}{|c|}{ Gram positive bacteria } & \multicolumn{2}{|c|}{ Gram negative bacteria } \\
\hline & $\begin{array}{c}\text { A. } \\
\text { fumigatus }\end{array}$ & $\begin{array}{c}P . \\
\text { italicum }\end{array}$ & $\begin{array}{c}C . \\
\text { albicans }\end{array}$ & $\begin{array}{c}\text { G. } \\
\text { candidum }\end{array}$ & $\begin{array}{c}S . \\
\text { aureus }\end{array}$ & $\begin{array}{c}B . \\
\text { subtilis }\end{array}$ & $\begin{array}{c}P . \\
\text { aeruginosa }\end{array}$ & E. coli \\
\hline $4 a$ & 125 & 125 & N.A. & 31.25 & 62.5 & 15.63 & N.A. & 500 \\
\hline $4 c$ & 31.3 & 15.6 & N.A. & 3.9 & 1.95 & 0.12 & N.A. & 62.5 \\
\hline $7 c$ & 31.3 & 15.6 & N.A. & 7.81 & 62.5 & 31.3 & N.A. & 500 \\
\hline $8 b$ & 15.6 & 7.8 & N.A. & 0.12 & 1.95 & 0.06 & N.A. & 31.3 \\
\hline $8 c$ & 125 & 62.5 & N.A. & 7.81 & 15.6 & 7.8 & N.A. & 500 \\
\hline $9 \mathbf{a}$ & 14.1 & N.A. & N.A. & 0.11 & 1.75 & 0.12 & N.A. & 26.5 \\
\hline $9 b$ & 0.9 & 15.3 & N.A. & 0.08 & 1.95 & 8.5 & N.A. & 500 \\
\hline Amphotericin B & 0.49 & 1.95 & 15.63 & 0.015 & N.A. & N.A. & N.A. & N.A. \\
\hline Ampicillin & N.A. & N.A. & N.A. & N.A. & 0.02 & 0.007 & N.A. & N.A. \\
\hline Gentamicin & N.A. & N.A. & N.A. & N.A. & N.A. & N.A. & 62.5 & 0.98 \\
\hline
\end{tabular}

\section{Experimental}

\subsection{General}

Melting points were determined using an electrothermal Gallenkamp apparatus and are reported uncorrected. IR spectra were recorded in KBr using a Pye Unicam SP-1000 Spectrometer. ${ }^{1} \mathrm{H}-\mathrm{NMR}$ spectra were recorded using DMSO- $\mathrm{d}_{6}$ solutions on a Varian EM-300 MHz Spectrometer and chemical shifts are reported in ppm relative to that of TMS, which was used as an internal standard. Mass spectra were recorded using a AEI MS 30 mass spectrometer operating at $70 \mathrm{eV}$. Elemental analyses were carried out by using the Microanalytical Center of Cairo University, Giza, Egypt. The enaminones 1a-d were prepared as previously reported [11]. ${ }^{13} \mathrm{C}-\mathrm{NMR}$ of compounds $\mathbf{7 d}, \mathbf{8 d}, \mathbf{9 c}$ and 9d could not be recorded due to the fact they precipitated in DMSO.

\subsection{Reaction of Enaminones 1a-d with Hydrazonoyl Chloride $\mathbf{2}$}

To a stirred solution of the appropriate enaminones $\mathbf{1 a}-\mathbf{d}(2.5 \mathrm{mmol})$ and the hydrazonoyl chloride 2 $(0.49 \mathrm{~g}, 2.5 \mathrm{mmol})$ in dry dioxane $(30 \mathrm{~mL})$, was added triethylamine $(0.5 \mathrm{~mL})$, and the mixture was heated for $5 \mathrm{~h}$. The precipitated triethylamine hydrochloride was filtered off, the filtrate was concentrated under reduced pressure, and the residue was triturated with methanol. The solid product so formed in each case, was collected by filtration, washed with water, dried, and recrystallized from 
ethanol to afford the corresponding 1,3,4-thiadiazole derivatives 4 . The products 4a-d prepared are listed below together with their physical constants.

5-[3-Acetyl-1-phenyl-1H-pyrazole-4-carbonyl]-2-benzoylimino-3-(4-methoxyphenyl)-3H-[1,3,4]thiadiazole (4a). Yellowish-red solid (81\% yield), mp 160-162 ${ }^{\circ} \mathrm{C}$; IR (KBr) $v_{\max } 1685,1642,1609$ (3C=O), 1554 $(\mathrm{C}=\mathrm{N}) \mathrm{cm}^{-1} ;{ }^{1} \mathrm{H}-\mathrm{NMR}\left(\mathrm{DMSO}_{6}\right) \delta 2.38\left(\mathrm{~s}, 3 \mathrm{H}, \mathrm{COCH}_{3}\right), 3.86\left(\mathrm{~s}, 3 \mathrm{H}, \mathrm{OCH}_{3}\right), 7.02-7.48(\mathrm{~m}, 5 \mathrm{H}, \mathrm{Ar}-\mathrm{H})$, 7.50-7.63 (m, 5H, Ar-H) 7.87 (d, $J=8 \mathrm{~Hz}, 2 \mathrm{H}$, Ar-H), 8.11 (d, $J=8 \mathrm{~Hz}, 2 \mathrm{H}$, Ar-H), 9.31 (s, 1H, pyrazolyl-H); ${ }^{13} \mathrm{C}-\mathrm{NMR}\left(\mathrm{DMSO}_{\mathrm{d}}\right.$ ) $\delta: 25.32,55.48,106.16,114.39,115.55,115.77,120.31,126.46$, $127.34,128.42$, 129.63, 132.50, 146.20, 148.56, 155.81, 155.86, 160.29, 162.02, 176.20, 190.21, 206.60. MS m/z (\%) $524\left(\mathrm{M}^{+}+1,2\right), 523\left(\mathrm{M}^{+}, 2\right), 408$ (7), 325 (2), 285 (2), 213 (10), 105 (24), 98 (100), 77 (27). Anal. Calcd. for $\mathrm{C}_{28} \mathrm{H}_{21} \mathrm{~N}_{5} \mathrm{O}_{4} \mathrm{~S}$ (523.56): C, 64.23; H, 4.04; N, 13.38. Found: C, 64.44; $\mathrm{H}, 4.17 ; \mathrm{N}, 13.58 \%$.

5-[3-Acetyl-1-phenyl-1H-pyrazole-4-carbonyl]-2-benzoylimino-3-(4-methylphenyl)-3H-[1,3,4]thiadiazole (4b). Yellowish-red solid (82\% yield), $\mathrm{mp}>300{ }^{\circ} \mathrm{C}$; IR (KBr) $v_{\max }, 1642,1617,1610(3 \mathrm{C}=\mathrm{O}), 1559$ $(\mathrm{C}=\mathrm{N}) \mathrm{cm}^{-1} ;{ }^{1} \mathrm{H}-\mathrm{NMR}\left(\mathrm{DMSO}_{6}\right) \delta 2.44\left(\mathrm{~s}, 3 \mathrm{H}, \mathrm{COCH}_{3}\right), 2.49$ (s, 3H, $\left.\mathrm{CH}_{3}-\mathrm{Ar}\right), 7.44$ (d, $J=8 \mathrm{~Hz}$, 2H, Ar-H), 7.47-7.51 (m, 5H, Ar-H), 7.52 (d, $J=8$ Hz, 2H, Ar-H), 7.61-8.11 (m, 5H, Ar-H), 9.32 (s, $1 \mathrm{H}$, pyrazolyl-H); ${ }^{13} \mathrm{C}-\mathrm{NMR}\left(\mathrm{DMSO}_{\mathrm{d}}\right) \delta$ : 11.58, 21.53, 114.0, 116.35, 120.48, 124.46, 126.91, $129.32,129.95,131.40,132.03,133.19,136.13,137.38,143.38,144.17,150.98,160.34,174.32$, 189.95, 196.30. MS m/z (\%) $508\left(\mathrm{M}^{+}+1,9\right), 507\left(\mathrm{M}^{+}, 15\right), 392$ (11), 213 (57), 121 (11), 105 (46), 98 (100), 77 (43). Anal. Calcd. for $\mathrm{C}_{28} \mathrm{H}_{21} \mathrm{~N}_{5} \mathrm{O}_{3} \mathrm{~S}$ (507.56): C, 66.26; H, 4.17; N, 13.80. Found: C, 66.39; $\mathrm{H}, 4.34 ; \mathrm{N}, 13.94 \%$.

5-[3-Acetyl-1-phenyl-1H-pyrazole-4-carbonyl]-2-benzoylimino-3-(4-chlorophenyl)-3H-[1,3,4]thiadiazole (4c). Yellow solid (80\% yield), mp 158-160 ${ }^{\circ} \mathrm{C}$; IR (KBr) $v_{\max } 1689,1638,1610(3 \mathrm{C}=\mathrm{O}), 1546(\mathrm{C}=\mathrm{N})$ $\mathrm{cm}^{-1}$; ${ }^{1} \mathrm{H}-\mathrm{NMR}\left(\mathrm{DMSO}_{\mathrm{d}}\right.$ ) $\delta 2.38\left(\mathrm{~s}, 3 \mathrm{H}, \mathrm{COCH}_{3}\right), 7.63-7.69$ (m, 5H, Ar-H), 7.89-8.15 (m, 5H, Ar-H) 7.43 (d, $J=8 \mathrm{~Hz}, 2 \mathrm{H}, \mathrm{Ar}-\mathrm{H}), 7.49$ (d, $J=8 \mathrm{~Hz}, 2 \mathrm{H}, \mathrm{Ar}-\mathrm{H}), 9.34$ (s, 1H, pyrazolyl-H); ${ }^{13} \mathrm{C}-\mathrm{NMR}$ $\left(\right.$ DMSO-d $\left._{6}\right) \delta: 21.45,112.64,115.31,119.72,122.22,126.15,126.80,127.78,128.40,128.81,131.20$, 132.58, 135.70, 138.47, 143.71, 151.17, 162.0, 176.06, 190.30, 206.03. MS $m / z(\%) 528\left(\mathrm{M}^{+}+1,2\right)$, $527\left(\mathrm{M}^{+}, 8\right), 412$ (4), 312 (36), $111(6), 105$ (45), 98 (100), 77 (39). Anal. Calcd. for $\mathrm{C}_{27} \mathrm{H}_{18} \mathrm{ClN}_{5} \mathrm{O}_{3} \mathrm{~S}$ (527.98): C, 61.42; H, 3.44; N, 13.26. Found: C, 61.62; H, 3.51; N, 13.45\%.

5-[3-Acetyl-1-phenyl-1H-pyrazole-4-carbonyl]-2-benzoylimino-3-(4-nitrophenyl)-3H-[1,3,4]thiadiazole (4d). Brown solid (78\% yield), mp 236-238 ${ }^{\circ} \mathrm{C}$; IR (KBr) $v_{\max } 1694,1636,1604(3 \mathrm{C}=\mathrm{O}), 1548(\mathrm{C}=\mathrm{N})$ $\mathrm{cm}^{-1}$; ${ }^{1} \mathrm{H}-\mathrm{NMR}\left(\mathrm{DMSO}_{6}\right) \delta 2.40\left(\mathrm{~s}, 3 \mathrm{H}, \mathrm{COCH}_{3}\right), 7.48-8.45$ (m, 14H, Ar-H), 9.54 (s, 1H, pyrazole H); ${ }^{13} \mathrm{C}-\mathrm{NMR}\left(\mathrm{DMSO}_{\mathrm{d}}\right.$ ) $\delta$ : 23.14, 113.24, 116.04, 119.56, 124.12, 126.26, 127.01, 127.71, 129.0, $129.21,131.0,132.69,136.35,138.17,142.08,152.11,159.90,172.14,190.17,201.37 . \mathrm{MS} \mathrm{m} / \mathrm{z}(\%)$ $539\left(\mathrm{M}^{+}+1,8\right), 538\left(\mathrm{M}^{+}, 4\right), 423$ (6), 406 (10), 356 (5), 292 (5), 105 (52), 98 (100), 77 (28). Anal. Calcd. for $\mathrm{C}_{27} \mathrm{H}_{18} \mathrm{~N}_{6} \mathrm{O}_{5} \mathrm{~S}$ (538.54): C, 60.22; H, 3.37; N, 15.61. Found: C, 60.05; H, 3.28; N, 15.39\%. 


\subsection{Reaction of Enaminones 1a-d with Heterocyclic Amines}

General procedure: To a solution of the appropriate enaminone $\mathbf{1 a}-\mathbf{d}(5 \mathrm{mmol})$ in acetic acid (20 $\mathrm{mL})$ was added the appropriate heterocyclic amine (3-aminotriazole or 2-aminobenzimidazole, $5 \mathrm{mmol}$ ). The mixture was stirred at reflux for $6 \mathrm{~h}$ then cooled. The formed solid was separated by filtration and recrystallized from dioxane to give compounds $7 \mathbf{a}-\mathbf{d}$ and $\mathbf{8 a}-\mathbf{d}$, respectively.

5-[2-Benzoylimino-3-(4-methoxyphenyl)-1,3,4-thiadiazol-5-yl]-[1,2,4]triazolo[1,5-a]pyrimidine (7a). Yellow solid (80\% yield), mp 235-236 ${ }^{\circ} \mathrm{C}$; IR (KBr) $v_{\max } 1604(\mathrm{C}=\mathrm{O}), 1558(\mathrm{C}=\mathrm{N}) \mathrm{cm}^{-1} ;{ }^{1} \mathrm{H}-\mathrm{NMR}$ $\left(\mathrm{DMSO}_{6}\right) \delta 3.88\left(\mathrm{~s}, 3 \mathrm{H}, \mathrm{OCH}_{3}\right), 7.48-7.59(\mathrm{~m}, 5 \mathrm{H}, \mathrm{Ar}-\mathrm{H}), 7.24(\mathrm{~d}, J=8 \mathrm{~Hz}, 2 \mathrm{H}, \mathrm{ArH}), 7.97$ (d, $J=8 \mathrm{~Hz}, 2 \mathrm{H}, \mathrm{Ar}-\mathrm{H}), 8.12$ (d, 1H, $J=4.5 \mathrm{~Hz}$, pyrimidinyl-H), 8.14 (d, $1 \mathrm{H}, J=4.5 \mathrm{~Hz}$, pyrimidinyl-H), 9.05 (s, 1H, triazolyl-H); ${ }^{13} \mathrm{C}-\mathrm{NMR}\left(\mathrm{DMSO}_{-} \mathrm{d}_{6}\right) \delta: 53.48,115.58,121.65,125.79,126.29,128.80$, $129.23,129.53,130.52,130.98,131.19,143.87,144.99,147.10,151.72,159.83,175.22 . \mathrm{MS} \mathrm{m} / \mathrm{z}(\%)$ $430\left(\mathrm{M}^{+}+1,6\right), 429\left(\mathrm{M}^{+}, 40\right), 352$ (3), 306 (26), 179 (2), 121 (43), 105 (100), 97 (8), 77 (66). Anal. Calcd. for $\mathrm{C}_{21} \mathrm{H}_{15} \mathrm{~N}_{7} \mathrm{O}_{2} \mathrm{~S}$ (429.46): C, 58.73; H, 3.52; N, 22.83. Found: C, 58.53; H, 3.38; N, 22.64\%.

5-[2-Benzoylimino-3-(4-methylphenyl)-1,3,4-thiadiazol-5-yl]-[1,2,4]triazolo[1,5-a]pyrimidine (7b). Yellow solid (79\% yield), mp > $300{ }^{\circ} \mathrm{C}$; IR $(\mathrm{KBr}) v_{\max } 1602(\mathrm{C}=\mathrm{O}), 1540(\mathrm{C}=\mathrm{N}) \mathrm{cm}^{-1}$; ${ }^{1} \mathrm{H}-\mathrm{NMR}$ $\left(\mathrm{DMSO}_{-} \mathrm{d}_{6}\right) \delta 2.46\left(\mathrm{~s}, 3 \mathrm{H}, \mathrm{CH}_{3}\right), 7.50-7.61(\mathrm{~m}, 5 \mathrm{H}, \mathrm{ArH}), 8.13(\mathrm{~d}, J=8 \mathrm{~Hz}, 2 \mathrm{H}, \mathrm{ArH}), 8.16(\mathrm{~d}, J=8 \mathrm{~Hz}$, 2H, ArH), $7.95(\mathrm{~d}, J=4.5 \mathrm{~Hz}, 1 \mathrm{H}$, pyrimidinyl-H), 8.97 (s, 1H, triazolyl-H), 9.05 (d, $J=4.5 \mathrm{~Hz}, 1 \mathrm{H}$, pyrimidinyl-H); ${ }^{13} \mathrm{C}-\mathrm{NMR}$ (DMSO-d ${ }_{6}$ ) $\delta$ : 18.27, 114.01, 120.24, 123.27, 127.06, 128.18, 129.05, $130.47,130.42,132.48,134.35,142.54,144.84,148.21,150.70,158.16,172.02 . \mathrm{MS} \mathrm{m} / z(\%) 414$ $\left(\mathrm{M}^{+}+1,3\right), 413\left(\mathrm{M}^{+}, 11\right), 306$ (10), 105 (100), 98 (2), 77 (62). Anal. Calcd. for $\mathrm{C}_{21} \mathrm{H}_{15} \mathrm{~N}_{7} \mathrm{OS}$ (413.46): C, 61.00; H, 3.66; N, 23.71. Found: C, 61.25; H, 3.47; N, 23.51\%.

5-[2-Benzoylimino-3-(4-chlorophenyl)-1,3,4-thiadiazol-5-yl]-[1,2,4]triazolo[1,5-a]pyrimidine (7c). Pale brown solid (76\% yield), mp $>300{ }^{\circ} \mathrm{C}$; IR $(\mathrm{KBr}) v_{\max } 1610(\mathrm{C}=\mathrm{O}), 1545(\mathrm{C}=\mathrm{N}) \mathrm{cm}^{-1}$; ${ }^{1} \mathrm{H}-\mathrm{NMR}$ $\left(\mathrm{DMSO}_{-} \mathrm{d}_{6}\right) \delta 7.38(\mathrm{~d}, J=8 \mathrm{~Hz}, 2 \mathrm{H}, \mathrm{ArH}), 7.49-7.96(\mathrm{~m}, 5 \mathrm{H}, \mathrm{ArH}), 8.12$ (d, $\left.J=8 \mathrm{~Hz}, 2 \mathrm{H}, \operatorname{ArH}\right), 8.24$ (d, $J=4.5 \mathrm{~Hz}, 1 \mathrm{H}$, pyrimidinyl-H), 8.45 (d, $J=4.5 \mathrm{~Hz}, 1 \mathrm{H}$, pyrimidinyl-H), 8.61 (s, $1 \mathrm{H}$, triazolyl-H); ${ }^{13}$ C-NMR (DMSO-d ${ }_{6}$ ) $\delta: 114.21,115.16,118.27,120.03,122.10,124.24,126.57,128.60,135.28$, 137.67, 142.11, 143.95, 147.09, 150.79, 155.20, 171.11. MS $m / z(\%) 435\left(\mathrm{M}^{+}+2,3\right), 434\left(\mathrm{M}^{+}+1,4\right)$, $433\left(\mathrm{M}^{+}, 9\right), 356$ (3), 306 (10), 105 (100), 98 (66), 77 (80). Anal. Calcd. for $\mathrm{C}_{20} \mathrm{H}_{12} \mathrm{ClN}_{7} \mathrm{OS}$ (433.87): C, 55.36; H, 2.79; N, 22.60. Found: C, 55.26; H, 2.64; N, 22.49\%.

5-(2-Benzoylimino-3-(4-nitrophenyl)-1,3,4-thiadiazol-5-yl)-[1,2,4]triazolo[1,5-a]pyrimidine

(7d). Brown solid (79\% yield), $\mathrm{mp}>300{ }^{\circ} \mathrm{C}$; IR $(\mathrm{KBr}) v_{\max } 1612(\mathrm{C}=\mathrm{O}), 1540(\mathrm{C}=\mathrm{N}) \mathrm{cm}^{-1}$; ${ }^{1} \mathrm{H}-\mathrm{NMR}$ $\left(\mathrm{DMSO}_{-} \mathrm{d}_{6}\right) \delta 7.53-7.64(\mathrm{~m}, 5 \mathrm{H}, \mathrm{ArH}), 8.26(\mathrm{~d}, J=8 \mathrm{~Hz}, 2 \mathrm{H}, \mathrm{ArH}), 8.52$ (d, $\left.J=8 \mathrm{~Hz}, 2 \mathrm{H}, \mathrm{ArH}\right), 8.56$ (d, $J=4.5 \mathrm{~Hz}, 1 \mathrm{H}$, pyrimidinyl-H), 8.59 (s, $1 \mathrm{H}$, triazolyl-H), $9.00(\mathrm{~d}, J=4.5 \mathrm{~Hz}, 1 \mathrm{H}$, pyrimidinyl-H); ${ }^{13} \mathrm{C}-\mathrm{NMR}$ (DMSO-d $\left.{ }_{6}\right) \delta$ : the sample precipitated. MS $m / z(\%) 444\left(\mathrm{M}^{+}, 17\right), 415$ (3), 367 (4), 306 (4), 299 (2), 121 (3), 105 (100), 90 (4), 77 (42). Anal. Calcd. for $\mathrm{C}_{20} \mathrm{H}_{12} \mathrm{~N}_{8} \mathrm{O}_{3} \mathrm{~S}$ (444.43): C, 54.05; H, 2.72; N, 25.21. Found: C, 54.18; H, 2.57; N, 25.05\%. 
4-[2-Benzoylimino-3-(4-methoxyphenyl)-1,3,4-thiadiazol-5-yl]-benzimidazo[1,2-a]pyrimidine

(8a).

Yellow solid (78\% yield), mp 265-266 ${ }^{\circ} \mathrm{C}$; IR $(\mathrm{KBr}) v_{\max } 1642(\mathrm{C}=\mathrm{O}), 1604(\mathrm{C}=\mathrm{N}) \mathrm{cm}^{-1}$; ${ }^{1} \mathrm{H}-\mathrm{NMR}$ $\left(\mathrm{DMSO}_{-} \mathrm{d}_{6}\right) \delta 3.88\left(\mathrm{~s}, 3 \mathrm{H}, \mathrm{OCH}_{3}\right), 7.17-7.59$ (m, 4H, Ar-H), 7.85 (d, J=7 Hz, 2H, Ar-H), 7.91-8.09 (m, 5H, Ar-H), 8.10 (d, $J=7 \mathrm{~Hz}, 2 \mathrm{H}, \operatorname{Ar}-\mathrm{H}), 8.14$ (d, $J=4.5 \mathrm{~Hz}, 1 \mathrm{H}$, pyrimidinyl-H), 9.68 (d, $J=4.5 \mathrm{~Hz}$, $1 \mathrm{H}$, pyrimidinyl-H); ${ }^{13} \mathrm{C}-\mathrm{NMR}\left(\mathrm{DMSO}_{\mathrm{d}}\right) \delta$ : 52.10, 112.70, 113.33, 115.37, 121.57, 121.77, 122.91, $125.85,126.38,126.52,130.67,133.72,134.74,135.08,135.48,137.24,144.36,148.74,149.50$, 150.80, 157.13, 169.26. MS m/z (\%) $479\left(\mathrm{M}^{+}+1,27\right), 478\left(\mathrm{M}^{+}, 29\right), 355$ (25), 194 (12), 121(10), 105 (83), 77 (98). Anal. Calcd. for $\mathrm{C}_{26} \mathrm{H}_{18} \mathrm{~N}_{6} \mathrm{O}_{2} \mathrm{~S}$ (478.53): C, 65.26; H, 3.79; N, 17.56. Found: C, 65.08; H, $3.56 ; \mathrm{N}, 17.33 \%$.

4-[2-Benzoylimino-3-(4-methylphenyl)-1,3,4-thiadiazol-5-yl]-benzimidazo[1,2-a]pyrimidine $(\mathbf{8 b})$. Orange solid (73\% yield), mp $270{ }^{\circ} \mathrm{C}$; IR ( KBr) $v_{\max } 1610(\mathrm{C}=\mathrm{O}), 1570(\mathrm{C}=\mathrm{N}) \mathrm{cm}^{-1}$; ${ }^{1} \mathrm{H}-\mathrm{NMR}$ $\left(\mathrm{DMSO}_{-} \mathrm{d}_{6}\right) \delta 2.35\left(\mathrm{~s}, 3 \mathrm{H}, \mathrm{CH}_{3}\right), 6.89-7.35$ (m, 4H, Ar-H), 7.45 (d, J=8 Hz, 2H, Ar-H), 7.74-8.01 (m, $5 \mathrm{H}, \operatorname{Ar}-\mathrm{H}), 8.0(\mathrm{~d}, J=8 \mathrm{~Hz}, 2 \mathrm{H}, \mathrm{Ar}-\mathrm{H}), 8.08(\mathrm{~d}, J=4.5 \mathrm{~Hz}, 1 \mathrm{H}$, pyrimidinyl-H), $9.24(\mathrm{~d}, J=4.5 \mathrm{~Hz}$, $1 \mathrm{H}$, pyrimidinyl-H); ${ }^{13} \mathrm{C}-\mathrm{NMR}\left(\mathrm{DMSO}_{\mathrm{d}}\right.$ ) $\delta: 14.15,115.63,116.0,121.22,122.77,125.49,126.02$, $128.01,128.51,128.59,129.27,129.82,130.04,131.50,134.74,136.68,139.23,140.82,142.08$, 152.37, 157.90, 168.95. MS m/z (\%) $462\left(\mathrm{M}^{+}, 18\right), 385$ (9), 355 (7), 285 (2), 195 (23), 105 (100), 77 (53). Anal. Calcd. for $\mathrm{C}_{26} \mathrm{H}_{18} \mathrm{~N}_{6} \mathrm{OS}$ (462.53): C, 67.52; H, 3.92; N, 18.17. Found: C, 67.40; H, 3.84; $\mathrm{N}, 18.27 \%$.

4-[2-Benzoylimino-3-(4-chlorophenyl)-1,3,4-thiadiazol-5-yl]-benzimidazo[1,2-a]pyrimidine $(\mathbf{8 c})$. Dark-orange solid (74\% yield), $\mathrm{mp}>300{ }^{\circ} \mathrm{C}$; IR $(\mathrm{KBr}) v_{\max } 1635(\mathrm{C}=\mathrm{O}), 1556(\mathrm{C}=\mathrm{N}) \mathrm{cm}^{-1} ;{ }^{1} \mathrm{H}-\mathrm{NMR}$ $\left(\right.$ DMSO-d $\left._{6}\right) \delta$ 7.47-7.64 (m, 4H, ArH), 7.85-8.15 (m, 5H, ArH), 7.71-7.80 (m, 4H, ArH), 8.37 (d, $J=4.5 \mathrm{~Hz}, 1 \mathrm{H}$, pyrimidinyl-H), $9.71\left(\mathrm{~d}, J=4.5 \mathrm{~Hz}, 1 \mathrm{H}\right.$, pyrimidinyl-H); ${ }^{13} \mathrm{C}-\mathrm{NMR}$ (DMSO-d 6 ) $\delta$ : $111.0,114.68,117.21,123.72,124.26,127.23,128.83,130.55,134.84,135.41,136.09,137.50$, 139.26, 145.20, 145.54, 147.70, 148.08, 149.24, 158.71, 159.67, 169.62. MS m/z (\%) $484\left(\mathrm{M}^{+}+2,13\right)$, $482\left(\mathrm{M}^{+}, 32\right), 397$ (10), 395 (8), 194 (22), 127 (18), 105 (100), 90 (49), 77 (71). Anal. Calcd. for $\mathrm{C}_{25} \mathrm{H}_{15} \mathrm{ClN}_{6} \mathrm{OS}$ (482.95): C, 62.17; H, 3.13; N, 17.40. Found: C, 62.30; H, 3.06; N, 17.30\%.

4-[2-Benzoylimino-3-(4-nitrophenyl)-1,3,4-thiadiazol-5-yl]-benzimidazo[1,2-a]pyrimidine (8d). Brown solid $\left(82 \%\right.$ yield), $\mathrm{mp}>300{ }^{\circ} \mathrm{C}$; IR $(\mathrm{KBr}) v_{\max } 1625(\mathrm{C}=\mathrm{O}), 1525(\mathrm{C}=\mathrm{N}) \mathrm{cm}^{-1} ;{ }^{1} \mathrm{H}-\mathrm{NMR}\left(\mathrm{DMSO}-\mathrm{d}_{6}\right) \delta$ 7.51-7.61(m, 5H, ArH), 7.86-7.95 (m, 4H, Ar-H), 8.15 (d, $J=5 \mathrm{~Hz}, 1 \mathrm{H}$, pyrimidinyl-H) 8.36-8.54 (m, 4H, ArH), 9.69 (d, $J=5 \mathrm{~Hz}, 1 \mathrm{H}$, pyrimidinyl-H); ${ }^{13} \mathrm{C}-\mathrm{NMR}$ (DMSO- $\mathrm{d}_{6}$ ) $\delta$ : the sample precipitated. MS m/z (\%) $493\left(\mathrm{M}^{+}, 1\right), 340$ (2), 122 (2), 105 (45), 98 (100), 77 (44), 55 (11). Anal. Calcd. for $\mathrm{C}_{25} \mathrm{H}_{15} \mathrm{~N}_{7} \mathrm{O}_{3} \mathrm{~S}$ (493.50): C, 60.84; H, 3.06; N, 19.87. Found: C, 60.65; H, 3.21; N, 19.70\%.

\subsection{Reaction of Enaminones 1a-d with Hydrazine Hydrate}

A mixture of the appropriate enaminones $1 \mathbf{a}-\mathbf{d}(5 \mathrm{mmol})$ and hydrazine hydrate $(5 \mathrm{~mL})$ in absolute ethanol was stirred at reflux for $10 \mathrm{~h}$ and cooled. The solid formed was separated by filtration and recrystallized from ethanol/dioxane mixture to give $\mathbf{9 a - d}$. 
N-[3-(4-Methoxyphenyl)-5-(1H-pyrazol-3-yl)-3H-[1,3,4]thiadiazol-2-ylidene]-benzamide (9a). White solid (83\% yield), mp 200-202 ${ }^{\circ} \mathrm{C}$; IR (KBr) $v_{\max } 3297(\mathrm{NH}), 1611(\mathrm{C}=\mathrm{O}), 1549(\mathrm{C}=\mathrm{N}) \mathrm{cm}^{-1}$; ${ }^{1} \mathrm{H}-\mathrm{NMR}\left(\mathrm{DMSO}_{6} \mathrm{~d}_{6}\right) \delta 3.69$ (s, 3H, $\mathrm{OCH}_{3}$ ), 7.08-7.11 (m, 9H, Ar-H), 7.88 (d, $J=8 \mathrm{~Hz}, 1 \mathrm{H}$, pyrazolyl-H), $7.95\left(\mathrm{~d}, J=8 \mathrm{~Hz}, 1 \mathrm{H}\right.$, pyrazolyl-H), 9.0 (s, 1H, NH); ${ }^{13} \mathrm{C}-\mathrm{NMR}$ (DMSO-d 6 ) $\delta: 56.12,114.31,116.09$, $119.11,121.64,125.32,125.32,128.08,128.47,129.10,136.20,142.62,154.03,160.12,170.13 . \mathrm{MS}$ m/z (\%) $377\left(\mathrm{M}^{+}, 2\right), 333$ (1), 288 (38), 266 (100), 251 (66), 179 (3), 149 (8), 133 (12), 121 (19), 104 (43), 98 (2), 77 (34). Anal. Calcd. for $\mathrm{C}_{19} \mathrm{H}_{15} \mathrm{~N}_{5} \mathrm{O}_{2} \mathrm{~S}$ (377.42): C, 60.46; H, 4.01; N, 18.56. Found: C, $60.49 ; \mathrm{H}, 4.11 ; \mathrm{N}, 18.29 \%$.

N-[3-(4-Methylphenyl)-5-(1H-pyrazol-3-yl)-3H-[1,3,4]thiadiazol-2-ylidene]-benzamide (9b). White solid, (86\% yield), mp 220-222 ${ }^{\circ} \mathrm{C}$; IR (KBr) $v_{\max } 3300(\mathrm{NH}), 1609(\mathrm{C}=\mathrm{O}), 1545(\mathrm{C}=\mathrm{N}) \mathrm{cm}^{-1}$; ${ }^{1} \mathrm{H}-\mathrm{NMR}\left(\mathrm{DMSO}_{6}\right) \delta 2.38\left(\mathrm{~s}, 3 \mathrm{H}, \mathrm{CH}_{3}\right), 7.04-7.06(\mathrm{~m}, 5 \mathrm{H}, \mathrm{Ar}-\mathrm{H}), 7.34-7.37(\mathrm{~m}, 4 \mathrm{H}, \mathrm{Ar}-\mathrm{H}) 7.46(\mathrm{~d}$, $J=8 \mathrm{~Hz}, 1 \mathrm{H}$, pyrazolyl-H), 7.88 (d, $J=8 \mathrm{~Hz}, 1 \mathrm{H}$, pyrazolyl-H), 9.15 (s, $1 \mathrm{H}, \mathrm{NH}) ;{ }^{13} \mathrm{C}-\mathrm{NMR}$ (DMSO-d 6 ) $\delta: 15.62,114.28,117.11,120.0,121.45,124.13,126.27,128.99,129.27,130.58,135.24,144.17$, 152.07, 158.08, 171.22. MS m/z (\%) $361\left(\mathrm{M}^{+}, 2\right), 272$ (3), 250 (61), 225 (4), 146 (5), 104 (24), 91 (20), 77 (16), 65 (14), 46 (100). Anal. Calcd. for $\mathrm{C}_{19} \mathrm{H}_{15} \mathrm{~N}_{5} \mathrm{OS}$ (361.42): C, 63.14; H, 4.18; N, 19.38. Found: C, $63.0 ; \mathrm{H}, 4.08 ; \mathrm{N}, 19.09 \%$.

$\mathrm{N}$-[3-(4-Chlorophenyl)-5-(1H-pyrazol-3-yl)-3H-[1,3,4]thiadiazol-2-ylidene]-benzamide (9c). Pale yellow solid $\left(78 \%\right.$ yield), mp $278-280{ }^{\circ} \mathrm{C}$; IR $(\mathrm{KBr}) v_{\max } 3315(\mathrm{NH}), 1607(\mathrm{C}=\mathrm{O}), 1546(\mathrm{C}=\mathrm{N}) \mathrm{cm}^{-1} ;{ }^{1} \mathrm{H}-\mathrm{NMR}$ $\left(\right.$ DMSO-d $\left._{6}\right) \delta 7.05-7.67(\mathrm{~m}, 9 \mathrm{H}, \mathrm{Ar}-\mathrm{H}), 7.74(\mathrm{~d}, J=8 \mathrm{~Hz}, 1 \mathrm{H}$, pyrazolyl-H), $8.28(\mathrm{~d}, J=8 \mathrm{~Hz}, 1 \mathrm{H}$, pyrazolyl-H), $10.22(\mathrm{~s}, 1 \mathrm{H}, \mathrm{NH}) ;{ }^{13} \mathrm{C}-\mathrm{NMR}\left(\mathrm{DMSO}-\mathrm{d}_{6}\right) \delta$ : the sample precipitated. MS $\mathrm{m} / \mathrm{z}(\%) 383$ $\left(\mathrm{M}^{+}+2,2\right), 382\left(\mathrm{M}^{+} 1,1\right), 381\left(\mathrm{M}^{+}, 5\right), 272$ (22), 271 (20), 270 (58), 192 (21), 105 (21), 111(52), 77 (100). Anal. Calcd. for $\mathrm{C}_{18} \mathrm{H}_{12} \mathrm{ClN}_{5} \mathrm{OS}$ (381.84): C, 56.62; H, 3.17; N, 18.34. Found: C, 56.48; H, 3.04; $\mathrm{N}, 18.25 \%$.

N-[3-(4-Nitrophenyl)-5-(1H-pyrazol-3-yl)-3H-[1,3,4]thiadiazol-2-ylidene]-benzamide (9d). Brown solid (81\% yield), mp 280-282 ${ }^{\circ} \mathrm{C}$; IR (KBr) $v_{\max } 3302(\mathrm{NH}), 1605(\mathrm{C}=\mathrm{O}), 1551(\mathrm{C}=\mathrm{N}) \mathrm{cm}^{-1}$; ${ }^{1} \mathrm{H}-\mathrm{NMR}\left(\mathrm{DMSO}_{\mathrm{d}}\right) \delta$ 7.51-7.58 (m, 5H, Ar-H), 7.73-7.77 (m, 4H, Ar-H) $7.86(\mathrm{~d}, J=8 \mathrm{~Hz}, 1 \mathrm{H}$, pyrazolyl-H), 8.19 (d, $J=8 \mathrm{~Hz}, 1 \mathrm{H}$, pyrazolyl-H), 10.34 (s, $1 \mathrm{H}, \mathrm{NH}) ;{ }^{13} \mathrm{C}-\mathrm{NMR}\left(\mathrm{DMSO}-\mathrm{d}_{6}\right) \delta$ : the sample precipitated. MS m/z (\%) $392\left(\mathrm{M}^{+}, 20\right), 298$ (7), 297 (5), 295 (5), 281 (100), 251 (67), 235 (11), 149 (21), 132 (44), 118 (24), 104 (78), 90 (25), 77 (71). Anal. Calcd. for $\mathrm{C}_{18} \mathrm{H}_{12} \mathrm{~N}_{6} \mathrm{O}_{3} \mathrm{~S}$ (392.39): C, 55.10; H, 3.08; N, 21.42. Found: C, 55.0; H, 3.21; N, 21.20\%.

\subsection{Microbiological Studies}

\subsubsection{Agar Diffusion Well Method to Determine the Antimicrobial Activity}

The microorganism inoculums were uniformly spread using sterile cotton swabs on a sterile Petri dish containing malt extract agar (for fungi) and nutrient agar (for bacteria). Each sample (100 $\mu \mathrm{L})$ was added to each well (6 $\mathrm{mm}$ diameter holes cut in the agar gel, $20 \mathrm{~mm}$ apart from one another). The systems were incubated for $24-48 \mathrm{~h}$ at $37{ }^{\circ} \mathrm{C}$ (for bacteria) and at $28{ }^{\circ} \mathrm{C}$ (for fungi). After incubation, microorganism growth was observed. Inhibition of the bacterial and fungal growth were measured in $\mathrm{mm}$. Tests were performed in triplicate [22]. 


\subsubsection{Minimal Inhibitory Concentration (MIC) Measurement}

The bacteriostatic activity of the active compounds (having inhibition zones (IZ) $\geq 16 \mathrm{~mm}$ ) was then evaluated using the two fold serial dilution technique. Two fold serial dilutions of the tested compounds solutions were prepared using the proper nutrient broth. The final concentration of the solutions was $132 ; 66 ; 33 ; 16.5$; and $8.25 \mathrm{mg} / \mathrm{mL}$. The tubes were then inoculated with the test organisms, grown in their suitable broth at $37^{\circ} \mathrm{C}$ for $24 \mathrm{~h}$ for bacteria (about $1 \times 10^{8} \mathrm{CFU} / \mathrm{mL}$ ), each $5 \mathrm{~mL}$ received $0.1 \mathrm{~mL}$ of the above inoculum and incubated at $37{ }^{\circ} \mathrm{C}$ for $24 \mathrm{~h}$. The lowest concentration showing no growth was taken as the minimum inhibitory concentration (MIC).

\section{Conclusions}

New series of 1,3,4-thiadiazoles incorporating pyrazole, triazolopyrimidine and benzimidazopyrimidines were synthesized via reaction of 1,3,4-thiadiazolenaminones with hydrazonoyl chloride and nitrogen nucleophiles. The structure of the new products was established based on elemental and spectral analysis. The antimicrobial activity results of the products indicated that some of the newly synthesized compounds showed promising activity.

\section{References}

1. Svete, J. Utilisation of chiral enaminones and azomethine imines in the synthesis of functionalised pyrazoles. ARKIVOC 2006, vii, 35-56.

2. Abushanab, F.A.; Sherif, S.M.; Mousa, S.A.S. Dimethylformamide dimethyl acetal as a building block in heterocyclic synthesis. J. Heterocycl. Chem. 2009, 45, 801-827.

3. Shawali, A.S.; Farghaly, T.A.; Al-Dahshoury, A.R. Synthesis, reactions and antitumor activity of new $\beta$-aminovinyl 3-pyrazolyl ketones. ARKIVOC 2009, xiv, 88-99.

4. Palmieri, G.; Cimarelli, C. Chemo- and stereoselective reduction of enaminones for the preparation of biologically active compounds. ARKIVOC 2006, vi, 104-126.

5. Al-Mousawi, S.; John, E.; Al-Kandery, N. Studies with enaminones: Synthesis and chemical reactivity of 2-(4-dimethylamino-2-oxobut-3-enyl)-isoindole-1,3-dione and of 4-(4-dimethylamino-2-oxobut-3-enyloxy)-2H-phthalazin-1-one. J. Heterocycl. Chem. 2004, 41, 381-385.

6. Li, Z.; Wang, X.; Da, Y. Synthesis of 2-(5-(2-chlorophenyl)-2-furoylamino)-5-aryloxymethyl1,3,4-thiadiazoles under microwave irradiation. Synth. Commun. 2001, 31, 1829-1836.

7. Supuran, C.T.; Briganti, F.; Tilli, S.; Chegwidden, W.R.; Scozzafava, A. Carbonic anhydrase inhibitors: Sulfonamides as antitumor agents. Bioorg. Med. Chem. 2001, 9, 703-714.

8. Liu, X.; Shi, Y.; Ma, Y.; Zhang, C.; Dong, W.; Pan, L.; Wang, B.; Li, Z. Synthesis, antifungal activities and 3D-QSAR study of $N$-(5-substituted-1,3,4-thiadiazol-2-yl)cyclopropanecarboxamides. Eur. J. Med. Chem. 2009, 44, 2782-2786.

9. Deminbas, N.; Karaoglu, S.A.; Demirbas, A.; Sancak, K. Synthesis and antimicrobial activities of some new 1-(5-phenylamino-[1,3,4]thiadiazol-2-yl)methyl-5-oxo-[1,2,4]triazole and 1-(4-phenyl5-thioxo-[1,2,4]triazol-3-yl)methyl-5-oxo- [1,2,4]triazole derivatives. Eur. J. Med. Chem. 2004, 39, 793-804. 
10. Holla, B.S.; Poorjary, K.N.; Rao, B.S.; Shivananda, M.K. New bis-aminomercaptotriazoles and bis-triazolothiadiazoles as possible anticancer agents. Eur. J. Med. Chem. 2002, 37, 511-517.

11. Farghaly, T.A.; Abdallah, M.A.; Muhammad, Z.A. Synthesis and evaluation of the anti-microbial activity of new heterocycles containing the 1,3,4-thiadiazole moiety. Molecules 2011, 16, 10420-10432.

12. Farghaly, T.A.; Abdalla, M.M. Synthesis, tautomerism, antimicrobial, anti-HCV, anti-SSPE, antioxidant and antitumor activities of arylazobenzosuberones. Bioorg. Med. Chem. 2009, 17, 8012-8019.

13. Abdel Hafez, N.A.; Farghaly, T.A.; Al-Omar, M.A.; Abdalla, M.M. Synthesis of bioactive polyheterocyclic ring systems as 5 $\alpha$-reductase inhibitors. Eur. J. Med. Chem. 2010, 45, 4838-4844.

14. Riyadh, S.M.; Farghaly, T.A.; Abdallah, M.A.; Abdalla, M.M.; Abd El-Aziz, M.R. New pyrazoles incorporating pyrazolylpyrazole moiety: Synthesis, anti-HCV and antitumor activity. Eur. J. Med. Chem. 2010, 45, 1042-1050.

15. Farghaly, T.A.; Abdel Hafez, N.A.; Ragab, E.A.; Awad, H.M.; Abdalla, M.M. Synthesis, anti-HCV, antioxidant, and peroxynitrite inhibitory activity of fused benzosuberone derivatives. Eur. J. Med. Chem. 2010, 45, 492-500.

16. Farghaly, T.A.; Gomha, S.M.; Abbas, E.M.; Abdalla, M.M. Hydrazonoyl halides as precursors for new fused heterocycles of 5 $\alpha$-reductase inhibitors. Archiv der Pharmazie 2012, 345, 117-122.

17. Negri, G.; Kascheres, C.; Kascheres, A.J. Recent development in preparation reactivity and biological activity of enaminoketones and enaminothiones and their utilization to prepare heterocyclic compounds. J. Heterocycl. Chem. 2004, 41, 461-491.

18. Shawali, A.S.; Farghaly, T.A.; Al-Dahshoury, A.R. Efficient synthesis of functionalized 3-(hetaryl)pyrazoles. ARKIVOC 2010, ix, 19-30.

19. Behbehani, H.; Ibrahim, H.M. 4-Thiazolidinones in heterocyclic synthesis: synthesis of novel enaminones, azolopyrimidines and 2-arylimino-5-arylidene-4-thiazolidinones. Molecules 2012, $17,6362-6385$.

20. Ho, Y.W. 5-(1-Pyrrolyl)-2-phenylthieno[2,3-d]pyrimidine as building block in heterocyclic synthesis. J. Chin. Chem. Soc. 2007, 54, 1075-1085.

21. Wen, L.-R.; Wang, S.-W.; Li, M.; Yang, H.-Z. Reaction of enaminones with aminopyrazoles: Synthesis, structures and bioactivities of 7-aryl-3-cyano-2-substituted pyrazolo[1,5-a]pyrimidines. Chin. J. Chem. 2005, 23, 1231-1235.

22. Smania, J.A.; Monache, F.D.; Smania, E.F.A.; Cuneo, R.S. Antibacterial activity of steroidal compounds isolated from Ganoderma applanatum (Pers.) Pat. (Aphyllophoromycetideae) fruit body. Int. J. Med. Mushrooms 1999, 1, 325-330.

Sample Availability: Samples of the compounds 4, 7, 8 and 9 are available from the authors.

(C) 2012 by the authors; licensee MDPI, Basel, Switzerland. This article is an open access article distributed under the terms and conditions of the Creative Commons Attribution license (http://creativecommons.org/licenses/by/3.0/). 